\title{
The effects of shifts in magnitude of reward on runway performance in immature and adult rats
}

WILLIAM A. ROBERTS

VASSAR COLLEGE

Three groups of immature rats and three groups of adult rats were each trained for 20 preshift trials with a different quantity of reward: .1, .5, or $2.5 \mathrm{gm}$ of wet mash. On 25 postshift trials, each group was rewarded with $.5 \mathrm{gm}$. In preshift training, amount of reward significantly differentiated immature groups but not adult groups. On postshift trials, however, rewardvalues significantly differentiated adult groups but not immature groups.

A number of experiments have shown that shifts in magnitude of reward have disruptive effects on the behavior of adult rats (e.g., Crespi, 1942; Zeaman, (1949). In the runway, a downward shift in reward leads to a drop in running speed while an upward shift in reward leads to an increase in running speed. An important fact about these changes in response speed is that they typically involve at least a temporary overshooting of the performance asymptotes of control animals run to the shifted-to magnitude of reward throughout. Both emotional and perceptual hypotheses have been advanced to account for these effects. As one in a series of experiments investigating the ontogeny of rat learning, the major purpose of this experiment was to see whether changes in behavior similar to those occasioned by shifts in reward in adult rats would occur with immature rats. It was also of interest to see whether immature animals would run at different speeds for different magnitudes of preshift reward. In a previous experiment (Roberts, 1966), immature rats did not run at different speeds for different quantities of reward. Data was also taken with adult rats to serve as a basis of comparison with the immature rat results.

\section{Method}

The apparatus consisted of a starting box ( 5 by 9 by 6 in.), a runway ( 5 by 93 by 6 in.), and a goal-box ( 7 by 13.5 by 6 in.), all made of wood and painted grey with hinged Plexiglas tops. The goal-box was placed at a right angle to the end of the runway and was entered by a right turn. A guillotine door separated the starting box and runway. When this door was raised, a microswitch, mounted below the door, was opened starting a Standard Electric Timer. When $\mathrm{S}$ brokea beam of red light passing across the runway 84 in. from the starting box door, the timer stopped. Times were recorded in .01 sec. and transformed to logs. A horizontally sliding Plexiglas door, located $24.5 \mathrm{in}$. before the goal-box entrance, was closed after $S$ entered the goalbox to prevent retracing. The S's reward was contained in a glass dish placed at the rear of the goal-box.

The Ss were 30 male and female immature and 30 male adult albino rats of the Wistar strain. The im- mature animals were 25 days old and the adult animals were 180 days old on the first day of experimental training. The experiment was run with one trial per day. Immature Ss were given their daily trial 15-17 hr. food deprived and were allowed to eat hard pellets ad lib for $6 \mathrm{hr}$. $1 \mathrm{hr}$. after each day's training trials. Adult Ss were maintained at $85 \%$ of their ad lib weight and were fed their daily ration of hard pellets $1 \mathrm{hr}$. after their daily trials.

A three group design was used at each age ıeves. Both immature and adult Ss were divided into three matched groups of 10 Ss each on the basis of body weight. The three groups were designated .1, .5, and 2.5, and were rewarded in preshift training with $.1, .5$, and 2.5 gm of wet mash, respectively. After 20 preshift training trials, all three groups were rewarded with $.5 \mathrm{gm}$ of wet mash for 25 postshift trials. Prior to the first day of experimental training, two days of pretraining were given in which each $S$ was given one direct feeding in the goalbox each day with the appropriate amount of reward. Results

Two adult animals, one in Group .1 and one in Group .5 , were discarded from the experiment for viciousness and refusal to run, respectively. Thus, the data presented for these groups are based on Ns of 9 . The preshift and postshift curves plotted in blocks of three trials are presented for immature Ss in Fig. 1 and for adult Ss in Fig. 2. At both age levels, the preshift acquisition curves are negatively accelerated with response time being inversely related to amount of reward. In both sets of curves, the difference between groups .1 and .5 is greater than the difference between groups .5 and 2.5. Analysis of Variance applied to the immature rat curves shows significant Groups ( $F=4.85, \mathrm{df}=2 / 27$ ) and Blocks $(F=75.90, \mathrm{~d} f=6 / 162)$ effects and a nonsignificant

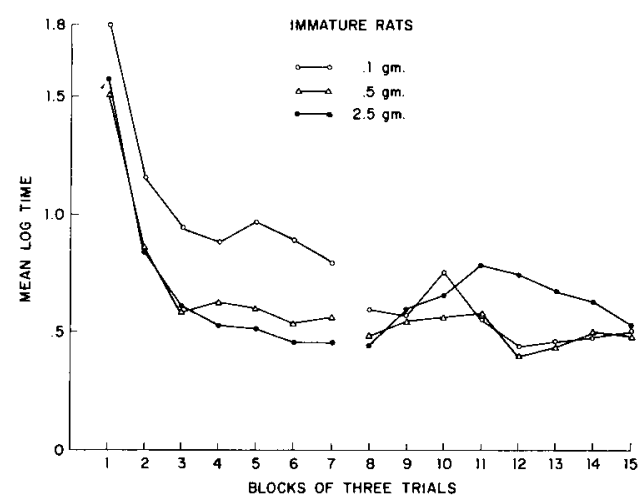

Fig. 1. Pre- and postshift performance curves for immature rats. 


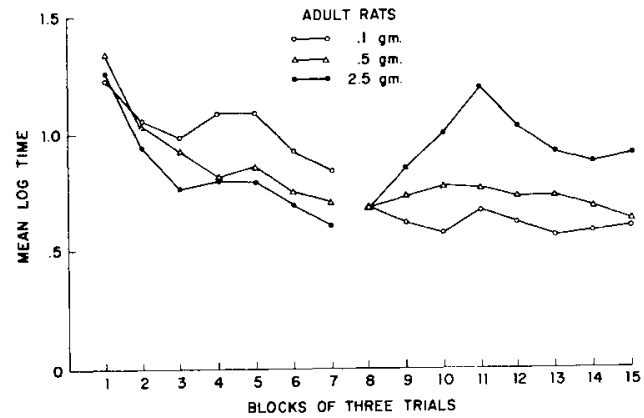

Fig. 2. Pre- and postshift performance curves for adult rats.

Groups by Blocks interaction $(F<1)$. Orthogonal comparisons reveal that the difference between groups .1 and .5 is significant $(F=5.91, d f=1 / 27)$, but the difference between groups .5 and 2.5 is not significant $(F<1)$. For the adult rat curves, the Groups effect is not significant $(F=1.97, \mathrm{df}=2 / 25)$ while the Blocks effect is significant $(F=47.82, \mathrm{df}=6 / 150)$ as is the Blocks by Groups interaction $(F=2.67, \mathrm{df}=12 / 150)$.

Examination of the postshift curves indicates that adult rats were more markedly affected by the shifts in reward magnitude than were the immature rats. For the adult animals, Group 2.5 rises steadily above Group .5 reaching a peak at Block 11 and subsequently declining to a level that is still above the control curve. Group .1 drops below Group .5 and remains there for all of the postshift blocks, although these curves appear to be merging by Block 15. The rise in Group 2.5 is more marked than the decline in Group .1.

The postshift curves for immature rats also demonstrate a rise in the response times of Group 2.5 which reaches a peak at Block 11 and then declines. This effect, however, is not as striking as that shown in the adult rat curves. The postshift curve for Group .1 declines over trials, but at no point does it show a tendency to undershoot the curve of Group .5. In fact, the curves for groups .1 and .5 are virtually identical over Blocks 11-15. Finally, the curves of all three groups appear to converge at Block 15.

Analysis of Variance on the postshift curves shows that the Groups effect was significant for adult animals ( $F=5.30, d f=2 / 25$ ), but the Groups effect did not approach significance for immature animals $(F<1)$. The Blocks effect is significant for immature Ss $(F=2.59, \mathrm{df}=7 / 189)$ and for adult $\mathrm{Ss}(\mathrm{F}=6.13 \mathrm{df}=7 / 175)$, and the Blocks by Groups interaction is significant for both immature rats $(\mathrm{F}=1.76, \mathrm{df}=14 / 189)$ and adult rats $(\mathrm{F}=3.91, \mathrm{df}=14 / 175)$. Orthogonal comparisons were made between the adult group means, and it was found that groups .5 and 2.5 are significantly different $(F=4.62$, $\mathrm{df}=1 / 25)$ while groups .1 and .5 are not $(\mathrm{F}<1)$.

\section{Discussion}

It is clear from the preshift results that magnitude of reward is inversely related to response time in the immature rat, at least when the reward values are .1 and $.5 \mathrm{gm}$ of wet mash. There are several possible reasons that this relationship did not appear in a previous experiment (Roberts, 1966). Dry pellets (45 mg) were used as reward in the previous experiment whereas wet mash was used here, and it may be that magnitude of reward is a more effective variable when the incentive is highly palatable. Secondly, the reward magnitudes used in the previous experiment were $5 \%$ and $25 \%$ of the animal's daily intake, and $5 \%$ of the daily intake was typically greater than 1 gm of food. Thus, the range of reward values used in the earlier experiment may have been one where speed of response is relatively insensitive to reward variation. It is surprising that magnitude of reward did not significantly differentiate adult groups in this experiment. It appears that with the reward parameters used in this experiment, immature Ss are more affected by preshift differences in reward than are adult Ss.

Clearly the opposite result holds in the postshift data. Here, shifts in reward magnitude had strong disruptive effects on adult behavior but relatively little effect on the performance of immature Ss. That the shifts in reward magnitude did have some effect on the immature animals is indicated by the significant Blocks by Groups interaction which shows that the group curves did change positions relative to one another over trials. These changes, however, were not strong enough to produce a significant overall groups difference.

This first exploratory study suggests that the "depression" and "elation" effects found with adult animals may not appear with immature animals. Such a finding would have important implications for the ontogenetic development of the processes responsible for these effects, be the processes emotional, perceptual, or both. Before such a conclusion is justified, more experiments must be done to look for these effects in the immature rat under a variety of experimental conditions. Variation of such parameters as drive level, degree of preshift training, and the reward shift ratio may well bring out significant "elation" and "depression" effects in the immature rat.

\section{References}

Crespi, L. P. Quantitative variation of incentive and performance in the white rat. Amer. J. Psychol., 1942, 55, 467-517.

Roberts, W. A. Learning and motivation in the immature rat. Amer. J. Psychol., 1966, in press.

Zeaman, D. Response latency as a function of the amount of reinforcement. J. exp. Psychol., 1949, 39, 466-483. 